\title{
Clinical effects and safety review of self-expanding stent surgery for extracranial carotid artery stenosis treatment
}

Q.F. Zhu, S.Z. Fang, G.F. Wang, Z.Z. Zhou, S.C. Bian, S.D. Cui, S.B. Yu, F.W. Wang, L.B. Shan and J. Kang

Department of Neurosurgery, PLA 264th Hospital, Taiyuan, China

Corresponding author: Q.F. Zhu

E-mail: qingfengzhucn@126.com

Genet. Mol. Res. 13 (3): 5128-5137 (2014)

Received May 24, 2013

Accepted October 3, 2013

Published July 7, 2014

DOI http://dx.doi.org/10.4238/2014.July.7.5

\begin{abstract}
This study aimed to investigate the clinical effects and safety review of self-expanding stent surgery in the treatment of extracranial carotid artery stenosis. Seventy-eight patients with carotid artery stenosis were applied with the self-expanding stent for endovascular interventional therapy. Eighty-one stents were implanted into 80 blood vessels of the 78 patients, in which protective umbrellas were used in 56 cases, and the success rate of stent implantation was $100 \%$. The stenosis degree decreased from the preoperative $(86.72 \pm 9.5 \%)$ to the postoperative $(13.43 \pm 5.62 \%)$ stage, and the blood peak velocity of the stenosed vessels decreased from 189.58 \pm 13.5 to $83.73 \pm 5.61 \mathrm{~cm} / \mathrm{s}$. Transient blood pressure and heart rate decreases occurred in 21 cases, continuously low blood pressure and heart rate decreasing occurred in 29 cases, and acute occlusion of the ipsilateral middle cerebral artery occurred in 1 case, which was resolved through thrombolysis and thrombus breaking in time. Overperfusion symptoms were observed in 13 cases, although without serious complications such as cerebral hemorrhage. The follow-up period continued for 6-32 months, and ultrasonography revealed that
\end{abstract}


77 cases had no stent-restenosis, while 1 case had restenosis. The application of self-expanding stents had good clinical effects, with fewer complications and higher safety for the treatment of extracranial carotid artery stenosis.

Key words: Carotid stenosis; Stent; Angioplasty; Angioplasty complication

\section{INTRODUCTION}

Stroke is the second ranking disease leading to death, and ischemic stroke is the main type of stroke, with the primary causes including extracranial carotid atherosclerosis, plaque formation, and vascular stenosis, etc. Once the stroke occurs, it causes high morbidity and mortality. Carotid artery stenosis is often the main reason of ischemic cerebrovascular disease. Drug treatments and surgical operations are the two most commonly used methods for the treatment of carotid stenosis. A number of large-scale, multi-centered, randomized clinically controlled trials have shown that among patients with symptomatic moderate carotid stenosis and asymptomatic severe stenosis, pure drug treatment was generally unsatisfactory in the latter patients, and that carotid endarterectomy (CEA), the standard surgery for carotid stenosis, would likely be more effective (Anonymous, 1991; Boules et al., 2005; Frego et al., 2009; Ederle et al., 2010; Plummer et al., 2011). In recent years, along with the developments of nerve interventional techniques and interventional materials science, the intervention of a percutaneous intravascular stent has become a research focus for the treatment of carotid artery stenosis, which has yielded very good results (Massop et al., 2009; Kan et al., 2012; Shobha et al., 2012). Although some studies have shown that carotid stent angioplasty may result in some complications, even severe complications, most researchers have suggested that the carotid stenting results are even better than CEA results (Tallarita et al., 2011; Fokkema et al., 2012; Dumont and Rughani, 2012). However, sufficient information about the long-term effects following carotid stenting are still lacking, and much remains unknown with respect to the safety and potential postoperative complications (Alvarez et al., 2012; Mohammadian et al., 2012). This study aimed to explore the surgical safety, complications, and clinical effects of self-expanding stents in treating carotid stenosis.

\section{MATERIAL AND METHODS}

\section{General information}

Seventy-eight cases were investigated, including 41 males ( 2 cases with severe bilateral carotid artery stenosis) and 37 females, aged from 44 to 87 years old with an average age of 72.5 years. Thirty-nine patients experienced repeated symptoms of transient ischemic attacks, 23 patients experienced influent speaking accompanied by limb weakness, 18 patients had symptoms of recurrent dizziness, 12 patients experienced personality changes and slow response, 6 patients experienced obvious memory impairment, while the remaining patients showed a mix of the above symptoms. Thirty-six cases had hypertension, 31 had diabetes, and 19 had coronary heart disease. The mean $( \pm$ SD) blood peak velocity of the 80 
stenosed vessels of the 78 patients was $189.58 \pm 13.5 \mathrm{~cm} / \mathrm{s}$. Digital subtraction angiogram (DSA) data was available for all patients, confirming that they suffered from extracranial carotid artery stenosis. The degree of stenosis was calculated based on the following formula: degree of stenosis $(\%)=$ stenosis-distal normal vessel diameter $(\mathrm{mm})$ - stenosis-section vessel diameter $(\mathrm{mm}) /$ stenosis-distal normal vessel diameter $(\mathrm{mm}) \times 100$.

This study was conducted in accordance with the Declaration of Helsinki and with approval from the Ethics Committee of the PLA 264th Hospital. Written informed consent was obtained from all participants.

\section{Selection of symptomatic indications}

Patients with extracranial carotid artery stenosis included in the study must have been suffering from symptomatic internal carotid artery stenosis $>50 \%$ or asymptomatic stenosis $>75 \%$, with current symptoms explained by vascular stenosis. Patients were excluded if a new stroke occurred within six weeks or if intracranial tumors or arteriovenous malformations were evident. The patients' cardiopulmonary and other vital organ functions had to be capable of withstanding surgical intervention. Patients included were those in which cerebral ischemic symptoms were not relieved or recurrent episodes occurred after regular medical drug treatments. In some cases, certain medication contraindications existed.

\section{Treatment}

The patients with the indications described above were orally administered $100 \mathrm{mg}$ aspirin per day and $75 \mathrm{mg}$ clopidogrel per day 3-5 days before the surgery. During the surgery, $2 \%$ lidocaine was used as local anesthesia, and then the Seldinger method was used to puncture the right femoral artery; an $8 \mathrm{~F}$ arterial sheath was placed with heparinization all over the body. The guiding catheter of the $8 \mathrm{~F}$ sheath was then guided to the proximal segment of the operative-side common carotid artery for visualization. The length and blood vessel diameter at the stenosis were measured again to select the appropriate embolic protection device, the sacculus was expanded, and the stent was applied. The stent was selected by referring to the diameters of both ends of the stenosis and the length of the stenosis; the stent diameter should be similar to the proximal vascular diameter of the stenosis in order to maintain good adherence. At the same time, the blood supplies of the ipsilateral anterior and middle cerebral artery were monitored. Guided by the road map, the Spider embolic protection device (EV3 Co., Ltd.) was applied to 56 patients in this study, which was directed into the siphon segment of carotid artery, passing the stenosis part, and was then continued along the intracranial direction. If the stenosis degree is greater than $50 \%$, different sacculus models should be used to expand the stenosis segment, which were applied to 48 patients. Later, the appropriate PROTEGERX self-expanding stents (EV3 Co., Ltd.) were selected and implanted at the desired position to cover the stenosis.

\section{Perioperative management}

Monitoring of postoperative vital signs was carried out for 24-48 h. Patients with hypertension were treated to adequate antihypertensive therapy, and received continuous 
intravenous micro-pump infusion of $10 \mathrm{mg}$ nitroglycerin $+50 \mathrm{~mL} 0.9 \%$ sodium chloride to maintain blood pressure at a level $2 / 3$ of the patients' basic blood pressure. This was done to avoid the possibility of excessively high blood pressure causing hyperperfusion into the original ischemic brain tissue, which would then lead to cerebral hemorrhage or excessive reperfusion injury. Because the stent might stimulate the carotid sinus baroreceptor, some patients might have suffered a drop in blood pressure and heart rate; in such cases, atropine and dopamine should be used to increase heart rate and blood pressure, respectively. One hundred milligrams aspirin (Bayer Schering Pharma; Aspirin Enteric-Coated Tabletsccds05-CNL, Chaner No. BJP2011056/47) per day and 75 mg clopidogrel (Sanofi Winthrop Industrie; Carbon Blanc Cedes, France) per day should be administrated postoperatively. After 3 months, the clopidogrel was stopped, whereas long-term use of $100 \mathrm{mg}$ aspirin once per day continued.

\section{Statistical analysis}

The degree of stenosis is reported as means \pm standard deviation (means \pm SD) using the statistical software package SPSS16.0. The preoperative and postoperative degrees of stenosis and blood flow velocity were carried out using the paired Student's $t$-test for comparisons, and $\mathrm{P}<0.05$ was considered to be statistically significant.

\section{RESULTS}

A total of 80 vessels were treated in 78 patients, including 2 cases of severe bilateral carotid artery stenosis, which were first treated on the main responsible vascular side, and then on the other side 3 weeks later. Eighty-one stents were implanted, of which 56 were accompanied with protective umbrellas, and 48 cases experienced sacculus pre-expansion before the release of the stent, who all received an embolic protection device. One case suffered severe internal carotid artery stenosis, and when the stent was placed, it hung in the carotid artery with poor adherence. Therefore, a slightly larger stent model was released inside the former stent, which showed good adherence. The success rate of stent placement was $100 \%$. The mean stenosis degree decreased from the preoperative $(86.72 \pm 9.5 \%)$ to the postoperative $(13.43 \pm 5.62 \%)$ stage, demonstrating that the degree of stenosis had significantly improved $(\mathrm{P}<0.01)$. The peak velocity of stenosis decreased from the preoperative $(189.58 \pm 13.5 \mathrm{~cm} / \mathrm{s})$ to the postoperative $(83.73 \pm 5.61 \mathrm{~cm} / \mathrm{s})$ stage $(\mathrm{P}<0.01)$. Twenty-one cases suffered from transient blood pressure and heart rate decreases, and 29 cases had persistent hypotension and decreased heart rates. One case (without umbrella) showed acute occlusion of the ipsilateral middle cerebral artery intraoperatively, which was resolved through emergent thrombolysis and thrombus breaking (Figure 1). The other 77 cases had no intraoperative complications of stroke. Thirteen cases showed perioperative hyperperfusion, although no brain bleeding or other serious complications occurred. Postoperative symptoms significantly improved, the follow-up period continued for 6-32 months, and carotid ultrasonography revealed no stent restenosis in 77 cases, whereas one patient had asymptomatic restenosis $(<50 \%)$, which was observed continuously during the follow-up period. A typical case is shown in Figure 2. 
A

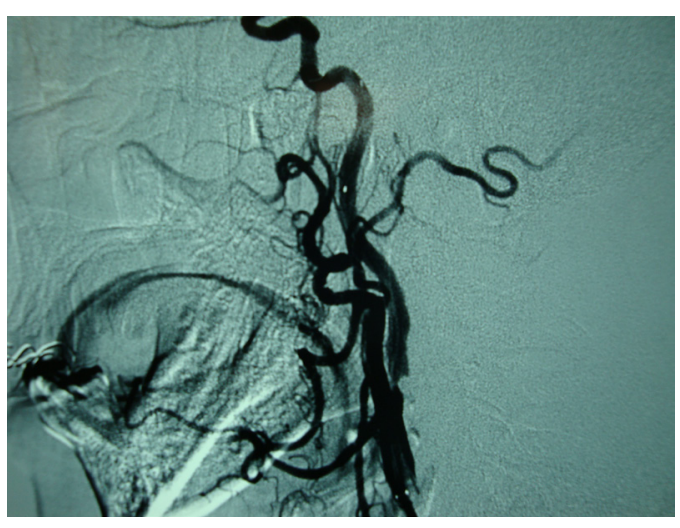

B

D

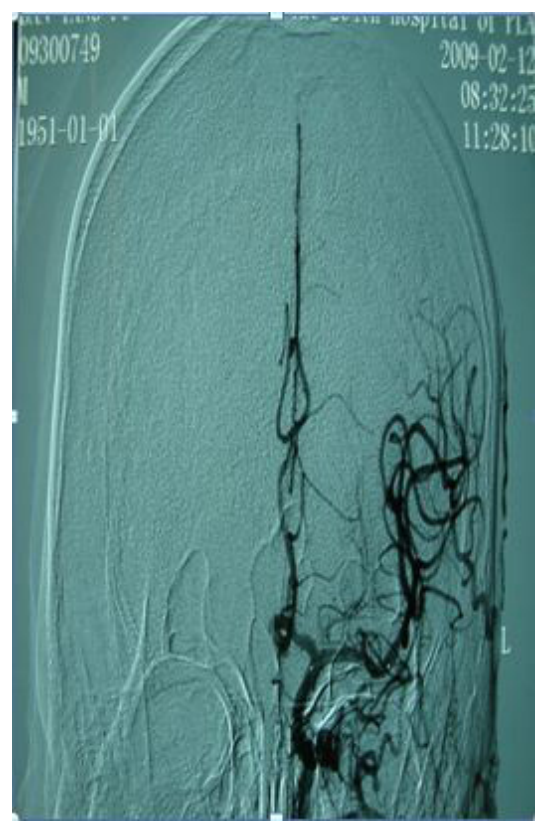

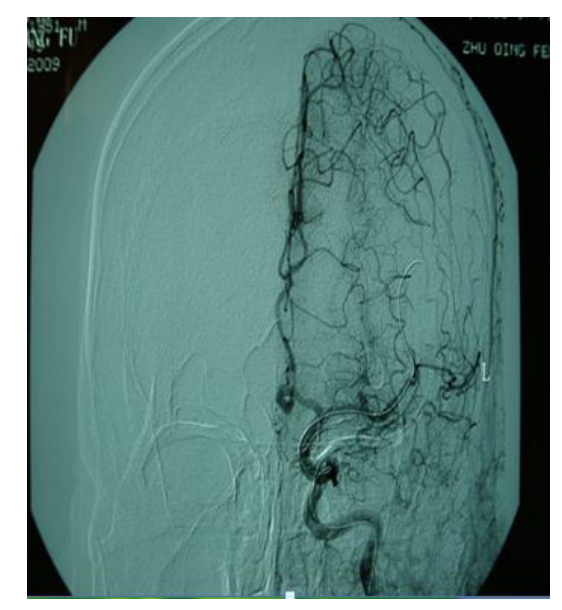

N
C

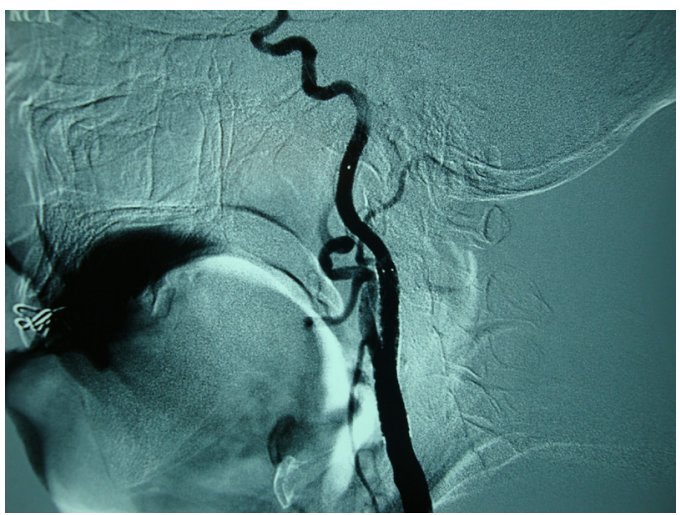


A

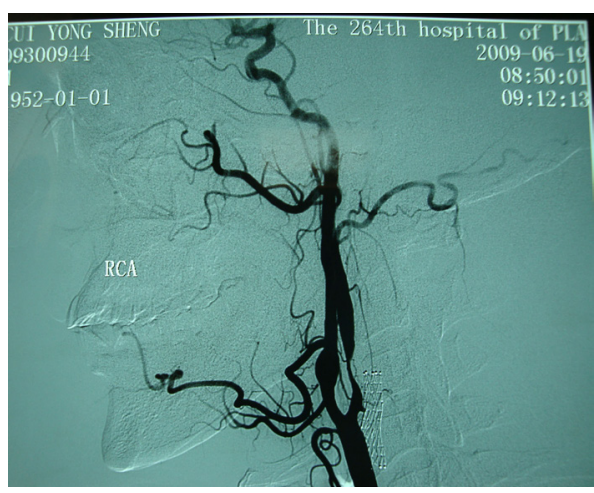

C

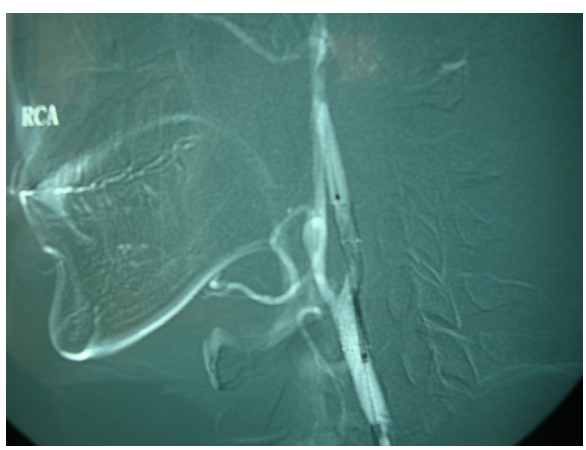

B

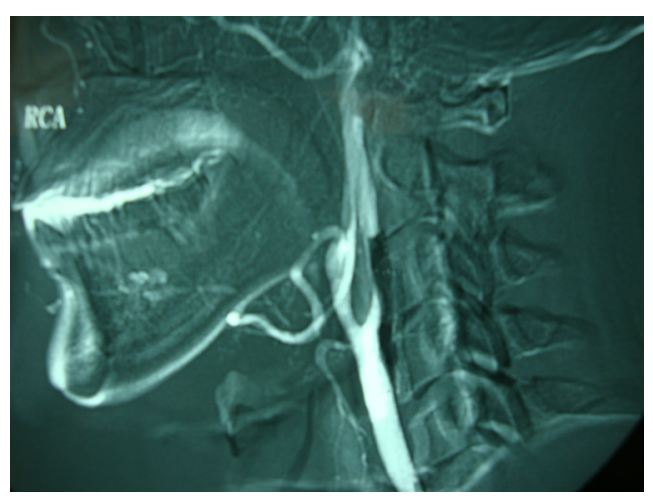

D

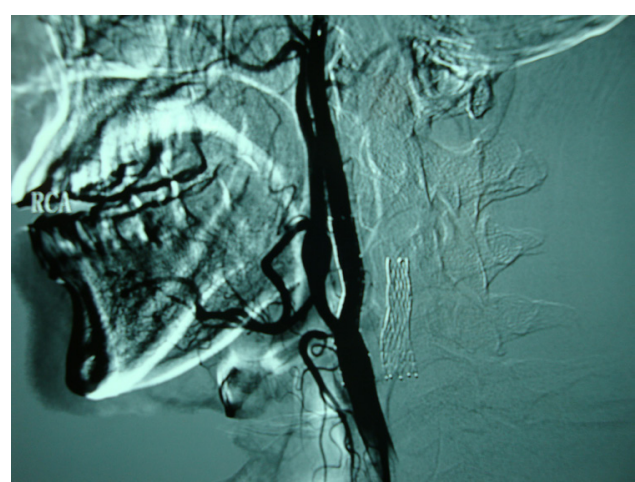

Figure 2. Patient 2, male, 69 years old, recurrent aphasia accompanied with the left side limb weakness. Digital subtraction angiogram showed the severe stenosis at the initial segment of the bilateral internal carotid artery. The carotid artery stenting was firstly performed on the right side, with no transient ischemic attacks recurred postoperatively, then stenosis stenting was performed on the left internal carotid artery side 3 weeks later. The hollow arrow in Figure 2A showed the severe stenosis in the left internal carotid artery, and the solid arrows showed the stent in the right common carotid artery; Figure 2B showed the sacculus pre-expansion on the stenosis spot; Figure 2C showed the relieving process of self-expanding stent, Figure 2D showed the left internal carotid artery stenosis completely returned back to normal after the stenting (hollow arrow).

\section{DISCUSSION}

Carotid artery stenosis is a direct risk factor of ischemic stroke, and is also the pathological basis of transient ischemic attacks (TIAs) or cerebral infarctions. CAE, along with medication, has been considered the standard treatment of carotid artery stenosis (Anonymous, 1991). With recent advances in materials science, especially the advent of cerebral protective devices and self-expanding stents, endovascular stents have become a research hotspot in the treatment of extracranial carotid artery stenosis in recent years (Kan et al., 2012; Dumont and Rughani, 2012), and it generally yields good results. Yadav et al. (2004) reported that the complication rate of stenting-angioplasty was $12.2 \%$, whereas the complication rate of CAE was $20.1 \%$, indicating that stenting was not inferior to endarterectomy. In this study, 
patients were all applied with laser engraved self-expanding stents (EV3 Co., Ltd.), with a $100 \%$ technical success rate of stent placement, vascular stenosis was immediately and significantly improved after the stenting, and the clinical symptoms also improved significantly. The long-term follow-up showed good results, with no serious perioperative complications. Nevertheless, the safety of carotid stenosis intravascular stent surgery has caused widespread concern, and further details need to be investigated in order to improve the clinical efficacy and safety of this treatment and reduce complications.

Stent migration is one of the major factors affecting the clinical efficiency of carotid stenosis stent angioplasty, which is more common when using the whole-weaving type of stent (Greil et al., 2003). Reports have indicated that the laser-carved stent only needed the first 2 to 3 stages for releasing, after which the stent could firmly anchor to the walls of blood vessels, and generally did not show situations of moving forward or backward (Alvarez et al., 2012). However, in the present study, the situation of stent-leaping forward did occur in one patient, leading to a too small stent residue in the carotid artery, and the stent end hung in the carotid artery with poor adherence and easy clot formation. Ultimately, a slightly bigger stent was released inside of the former stent, and together, the two overlaid stents achieved good adherence. One possible cause of this movement might be based on the patient's arteriosclerosis, coupled with a too small stent diameter, leading to the stent being not well anchored to the vessels. In addition, the proactive umbrella should have been secured after the stent was released, which would avoid the down-shifting as well as the stent-twisting. When collecting the umbrella, it must be completely recycled to the umbrella sheath; otherwise, the umbrella might become twisted with the stent, leading to migration of the stent when withdrawing the micro-guide wire and the protective devices of the stent.

Embolism falling is the most serious complication of carotid stenosis stent angioplasty, and plaque falling could cause cerebral embolisms, and even neurological dysfunction in severe situations. The main reason for this lies in the potentially violent intraoperative extrusion and cutting action for sacculus expansion and stent releasing toward the atherosclerotic plaques on the stenosed vessels, or due to the stimulation from the stent and guiding wire. In recent years, the use of cerebral protective devices significantly reduced the incidence of cerebral embolisms. Wholey et al. (2003) reported that in a global study of registered carotid stents, the mortality rate of 6753 cases without cerebral protective devices was $5.29 \%$, while it was only $2.23 \%$ in the 4221 cases with cerebral protective devices. In this study, the 56 cases with protective devices did not have cerebral embolic events, among which, visible lipid debris could be seen with the naked eye in 11 cases in the recovered umbrella. Among the 22 cases without protective umbrellas, in 1 case, acute occlusion occurred on the M2-segment of the ipsilateral middle cerebral artery when the sacculus was expanded. The patient felt right limb weakness accompanied with aphasia within $5 \mathrm{~min}$, and then gradually became confused. The blood vessel was re-opened after the thrombolysis and mechanical thrombus breaking was performed for $50 \mathrm{~min}$ (Figure 1), and the patient's neurological symptoms gradually improved. To reduce such complications in the future, the following steps are recommended. During the operation, the action must be gentle, careful, and accurate, and the guide wire technology must be flexibly used; when facing a patient with severe stenosis, a vessel protective device must be used, namely a protective umbrella, for sacculus expansion. The sacculus expansion should be carried out only once the umbrella is put in place, and the self-expanding stents could be released later. If the angiography reveals that residual stenosis is $<30 \%$ after the stenting, aboral sacculus expansion would generally not be recommended. This is because the 
stent mesh would cut the plaque during the aboral sacculus expansion, which might cause the falling of a large number of plaques (Sadek et al., 2009). Moreover, the memory effect of the stent would still be able to slowly expand the main responsible vessels after self-expanding.

The carotid sinus baroreceptors are located in the blood vessels of the carotid sinus region, and are easy to stimulate, causing tension elevation of the vagus nerve and a consequent drop in heart rate and blood pressure during the sacculus expansion or stent releasing procedures. Stimulation of the carotid sinus baroreceptors is more common at the exact moment of sacculus expanding in patients with severe stenosis, causing a rapid drop in the heart rate and blood pressure, and even cardiac arrest in some cases. In this study, 21 cases had transient decreases in blood pressure and heart rate or persistent hypotension, and decreasing heart rates occurred in 29 cases, in which atropine and dopamine were used to maintain normal heart rate and blood pressure. Atropine was used to increase the heart rate to 75 beats $/ \mathrm{min}$ or more before the sacculus expansion, while atropine and dopamine were prepared for possible use. If the heart rate falls to $<50$ beats/min or blood pressure declines sharply after the sacculus expansion, arterial injection should be performed through the guiding catheter, and then slow intravenous continuous infusion should be followed in order to maintain a normal heart rate and blood pressure. The patients in this study all recovered after 1 week of treatment. In addition, in order to reduce the continuous stimulation of the self-expanding stents toward the carotid sinus, the diameter of the stent should match that of the vessel. If the stent diameter were too large, continuous expansion would likely continuously stimulate the carotid sinus baroreceptor.

Hyperperfusion syndrome is a serious complication after interventional treatment of ischemic cerebral vessels, which is associated with postoperative pain in ipsilateral frontal or periorbital regions, cerebral hemorrhages in severe cases, and fatal complications (Gupta et al., 2005). Schaafsma et al. (2002) suggested that a blood-flow velocity level of the postoperative ipsilateral middle cerebral artery exceeding the preoperative level by $100 \%$ would be an early and sensitive indicator of the occurrence of hyperperfusion syndrome. Thirteen patients in this study showed perioperative transition perfusion, and they all had a high stenosis situation. Such patients' main blood supply vessels would be in a state of long-period hypoperfusion, and vascular autoregulation would be reduced when the other intracranial vascular compensation was poor. Once the stent is implanted, stenosis would improve, and the blood would largely rush in, resulting in a sudden increase in perfusion pressure and over-perfusion performance. No case of fatal complications, such as cerebral hemorrhage, occurred in this study as the patients' blood pressure was immediately controlled after stent implantation so that it could drop to $2 / 3$ of the basic level to effectively prevent the chance of cerebral hemorrhage due to the sudden increase in perfusion pressure. In addition, patients with bilateral carotid stenosis could not be simultaneously implanted with stents, or hyperperfusion syndrome would occur postoperatively. However, there is still no uniform standard for the interval between the operations. Gupta et al. (2005) suggested that an interval of 6 to 8 weeks was appropriate. In our study, 2 patients suffered from severe stenosis of the bilateral carotid artery (Figure 2), and the interval between operations was 3 weeks with no complications observed postoperatively.

Stent restenosis is one of the most important factors affecting the long-term effects of carotid stent angioplasty (Khan et al., 2003; Setacci et al., 2003; Gurm et al., 2008; Moreira et al., 2012). Restenosis is caused by the hyperplasia of the smooth muscle cells or endomembrane, rather than by the atherosclerosis plaque. In a systematic review of stenting for carotid stenosis, Groschel et al. (2005) found that the restenosis rate was about $4 \%$ in 2 years. In the present study, the follow-up period lasted more than 6 months, and only 1 restenosis $(1.22 \%)$ 
occurred in this time. This patient had approximately $56 \%$ preoperative stenosis, and during the surgery, the sacculus expansion was not used, but the self-expanding stent was instead simply released in the hope that the stent could play a role in the continuous expansion. However, the stent did not appear to facilitate follow-up expansion, causing a residual amount of the original stenosis to remain. This suggested that when plaques were hard, the subsequent expansion of the self-expanding stent would be limited. Therefore, the sacculus expansion must be performed before the stenting, and its self-expanding role cannot be relied upon. In addition, the administration of aspirin, clopidogrel, and statins would be an important measure in preventing stent restenosis.

In summary, the data showed that once important aspects are fully grasped, self-expanding stent angioplasty will have good and safe clinical effects for the treatment of extracranial carotid artery stenosis. However, this study only shows the results from a single center and the sample size was small. Therefore, these results await confirmation with randomized studies of larger samples from multiple centers.

\section{ACKNOWLEDGMENTS}

Research supported by the Scientific Key Project of Shanxi Province (Project \#20120313018-2).

\section{REFERENCES}

Anonymous (1991). North American Symptomatic Carotid Endarterectomy Trial. Methods, patient characteristics, and progress. Stroke 22: 711-720.

Alvarez B, Matas M, Ribo M, Maeso J, et al. (2012). Transcervical carotid stenting with flow reversal is a safe technique for high-risk patients older than 70 years. J. Vasc. Surg. 55: 978-984.

Boules TN, Proctor MC, Aref A, Upchurch GR Jr, et al. (2005). Carotid endarterectomy remains the standard of care, even in high-risk surgical patients. Ann. Surg. 241: 356-363.

Dumont TM and Rughani AI (2012). National trends in carotid artery revascularization surgery. J. Neurosurg. 116: 12511257.

Ederle J, Dobson J, Featherstone RL, Bonati LH, et al. (2010). Carotid artery stenting compared with endarterectomy in patients with symptomatic carotid stenosis (International Carotid Stenting Study): an interim analysis of a randomised controlled trial. Lancet 375: 985-997.

Fokkema M, den Hartog AG, Bots ML, van der Tweel I, et al. (2012). Stenting versus surgery in patients with carotid stenosis after previous cervical radiation therapy: systematic review and meta-analysis. Stroke 43: 793-801.

Frego M, Bridda A, Ruffolo C, Scarpa M, et al. (2009). The hostile neck does not increase the risk of carotid endarterectomy. J. Vasc. Surg. 50: 40-47.

Greil O, Pflugbeil G, Weigand K, Weiss W, et al. (2003). Changes in carotid artery flow velocities after stent implantation: a fluid dynamics study with laser Doppler anemometry. J. Endovasc. Ther. 10: 275-284.

Groschel K, Riecker A, Schulz JB, Ernemann U, et al. (2005). Systematic review of early recurrent stenosis after carotid angioplasty and stenting. Stroke 36: 367-373.

Gupta AK, Purkayastha S, Unnikrishnan M, Vattoth S, et al. (2005). Hyperperfusion syndrome after supraaortic vessel interventions and bypass surgery. J. Neuroradiol. 32: 352-358.

Gurm HS, Yadav JS, Fayad P, Katzen BT, et al. (2008). Long-term results of carotid stenting versus endarterectomy in high-risk patients. N. Engl. J. Med. 358: 1572-1579.

Kan P, Mokin M, Dumont TM, Snyder KV, et al. (2012). Cervical carotid artery stenosis: latest update on diagnosis and management. Curr. Probl. Cardiol. 37: 127-169.

Khan MA, Liu MW, Chio FL, Roubin GS, et al. (2003). Predictors of restenosis after successful carotid artery stenting. Am. J. Cardiol. 92: 895-897.

Massop D, Dave R, Metzger C, Bachinsky W, et al. (2009). Stenting and angioplasty with protection in patients at high-risk for endarterectomy: SAPPHIRE Worldwide Registry first 2,001 patients. Catheter. Cardiovasc. Interv. 73: 129-136. 
Mohammadian R, Sohrabi B, Mansourizadeh R, Mohammadian F, et al. (2012). Unprotected carotid artery stenting: complications in 6 months follow-up. Neuroradiology 54: 225-230.

Moreira T, Michel P, Binaghi S and Hirt L (2012). Risk factor impact on blood flow velocities and clinical outcomes of stented cervical and intracranial stenoses: preliminary observations. Clin. Neurol. Neurosurg. 114: 922-929.

Plummer C, Henderson RD, O'Sullivan JD and Read SJ (2011). Ischemic stroke and transient ischemic attack after head and neck radiotherapy: a review. Stroke 42: 2410-2418.

Sadek M, Cayne NS, Shin HJ, Turnbull IC, et al. (2009). Safety and efficacy of carotid angioplasty and stenting for radiation-associated carotid artery stenosis. J. Vasc. Surg. 6: 1308-1313.

Schaafsma A, Veen L and Vos JP (2002). Three cases of hyperperfusion syndrome identified by daily transcranial Doppler investigation after carotid surgery. Eur. J. Vasc. Endovasc. Surg. 23: 17-22.

Setacci C, Pula G, Baldi I, de Donato G, et al. (2003). Determinants of in-stent restenosis after carotid angioplasty: a casecontrol study. J. Endovasc. Ther. 10: 1031-1038.

Shobha N, Almekhlafi MA, Pandya A, Couillard PL, et al. (2012). Carotid angioplasty and stenting is safe in women. Can. Assoc. Radiol. J. 63: S18-S22.

Tallarita T, Oderich GS, Lanzino G, Cloft H, et al. (2011). Outcomes of carotid artery stenting versus historical surgical controls for radiation-induced carotid stenosis. J. Vasc. Surg. 53: 629-636.

Wholey MH, Al-Mubarek N and Wholey MH (2003). Updated review of the global carotid artery stent registry. Catheter. Cardiovasc. Interv. 60: 259-266.

Yadav JS, Wholey MH, Kuntz RE, Fayad P, et al. (2004). Protected carotid-artery stenting versus endarterectomy in highrisk patients. N. Engl. J. Med. 351: 1493-1501. 